\title{
Expression of Nodal on Bronchial Epithelial Cells Influenced by Lung Microbes Through DNA Methylation Modulates the Differentiation of T-Helper Cells
}

\author{
Lili Wang Guojun Wu Xiaoqun Qin Qiongshan Ma Yigang Zhou Shuiping Liu \\ Yurong Tan
}

Department of Microbiogy, Xiangya School of Medicine, Central South University, Changsha, China

\section{Key Words}

Airway hyperresponsiveness $•$ Human bronchial epithelial cells $\bullet$ T helper cells $•$ Nodal

\begin{abstract}
Background/Aims: The previous study in our lab showed that Nodal molecule on bronchial epithelial cells (BECs) was modulated by all kinds of lung microbes. The present study was designed to determine the effects of Nodal on proliferation of BECs and BECs-induced differentiation of T-helper (Th) cells. The epigenetic mechanisms of Nodal expression following treatments of different lung microbes were also identified. Methods: Real-time polymerization chain reaction (PCR) and western blot were used to determine the expression of Nodal. Flow cytometry was used to observe the effects of proliferation of BECs and subsequent BECsinduced differentiation of Th cells. Methylation levels of CpG islands in Nodal promoters were also analyzed by time of flight mass spectrometry. Results: The results showed that Nodal promoted proliferation of BECs and BECs-induced differentiation of Th cell from Th1 to Th2 and Th17. Nodal promoter showed a hyper-methylation in normal BECs. Through methylation modification in the promoter, $P$. aeruginosa or A.baumanni inhibited the expression of Nodal while RSV promoted the expression of Nodal. Conclusions: Our data showed that Nodal promoted Th2 and Th17 differentiation and inhibited Th1 differentiation which may cause imbalance of airway microenvironment. P. aeruginosa or A.baumanni may be hopeful for the treatment of airway hyperresponsveness by inhibition Nodal expression through DNA methylation modification in the promoter.
\end{abstract}

Copyright (C) 2015 S. Karger AG, Basel

\section{Introduction}

Nowadays, there are a lot of theories to explain the mechanisms of airway hyperresponsiveness (AHR) such as immune imbalance [1], neurogenic inflammation [2],

L. Wang and G. Wu made equal contributions to this work.

Shuiping Liu and Yurong Tan

Department of Microbiology, Xiangya School of Medicine, Central South University, Changsha 410078, Hunan, (China)

Tel.+8673182355003, E-Mail hope7@126.com 
airway infection and inflammation [3] and epithelial defect [4] and these theories explain the mechanisms and pathologies of AHR respectively from different perspectives, but cannot explain all the manifestations of AHR. Now, most scholars accept the opinion that diseases with AHR such as chronic bronchitis, asthma and COPD are chronic airway inflammation [5]. Chronic airway inflammation usually ascribes to bacterial or viral infection, or irritability of dusts or chemical substances. As the main targets of chronic inflammation, BECs may be the initiating factor for occurrence and development of AHR.

Lung microbiota refers to all kinds of microorganisms in the lungs. By using new technologies such as 16s rRNA and gene sequencing, the existence of the lung microbiota has been confirmed [6, 7]. In fact, epithelial cells are the main targets of various microorganisms, which may exert important influences on epithelial proliferation and wound repair. In a previous study, we observed that some microbes in the lungs have beneficial effects to the epithelia such as inactivated $P$. aeruginosa promoted proliferation of normal, OVA-stressed and respiratory syncytial virus (RSV)-infected BECs, reduced the expression of aging markers on BECs, inhibited airway inflammation and AHR, and promoted differentiation of Th1 cells and inhibited differentiation of Th2 and Th17 $[8,9]$. On the other hand, some microbes in the lungs may cause adverse influences to the epithelia. For example, nonstructural proteins of RSV can combine with histone H2BD specifically, and further induce ubiquitination of $\mathrm{H} 2 \mathrm{BD}$ and subsequent gene expression of HOXa5 and HOXb6, which may be associated with abnormal development of BECs and increased susceptibility to asthma [10].

In a previous unpublished data of study, we used a $\mathrm{RT}^{2}$ Profiler $^{\mathrm{TM}}$ PCR Array Human Epithelial to Mesenchymal Transition and observed that different lung microbes had different effects on the expression of Nodal molecule on BECs. For example, RSV promoted the mRNA expression of Nodal, while P. aeruginosa and A.baumanni inhibited the expression of Nodal. Nodal is a kind of secreted protein belonging to transforming growth factor (TGFbeta) superfamily. It is encoded by the nodal gene which locates on chromosome 10 q22. 1 and is one of the important genes which play a vital role during embryonic development [11]. In addition, Nodal seems to have important functions in neural patterning, stem cell maintenance and many other developmental processes $[12,13]$. Based on the studies of Nodal, it was shown that Nodal acts by binding to heteromeric complexes between type I and type II Activin receptors, which in turn acts through the Smad2/3 signaling pathway to the nucleus and promotes the expression of genes involved in proliferation and differentiation [14]. Nodal also further activates its own expression via a positive feedback loop [15]. Nodal signaling is tightly regulated by Cripto, an extracellular GPI-linked protein that acts as a cofactor, while inhibited by Lefty and Cerberus [16]. However, the function of Nodal in the adult human airway epithelia remains unclear. Moreover, Nodal is one of important genes during embryonic development, and remains a low level of expression in adults. High levels of expression are usually associated with many kinds of cancers such as prostate and pancreatic cancer $[17,18]$ and Lefty and Cerberus are hopeful to treat various cancers [19], indicating that methylation modification may get involved in the expression of Nodal.

So, in the present study, firstly, we used real-time PCR and western blotting to confirm the expression of Nodal influenced by different lung microbes. Then, we addressed the question of Nodal expression in the BECs on proliferation of BECs and subsequent BECsinduced differentiation of Th cells. The epigenetic mechanisms of Nodal expression following treatments of different lung microbes were also identified. Based on our results, we propose that bacterial components may be as a strategy for the treatment and prevention of AHR.

\section{Materials and Methods}

Reagents

P. aeruginosa, A.baumanni and RSV (strain A2) were preserved in our lab. RSV Real-time PCR Kit was purchased from Huayin Medicine Biotechnology Co. Ltd., Guangzhou, China. Monoclonal rabbit anti-Nodal, monoclonal mouse anti-NS1, anti-IL-4, anti-IL-17 and anti-IFN-gamma antibodies were from Abcam, USA. 


\section{Cellular Physiology Cell Physiol Biochem 2015;37:2012-2022 \begin{tabular}{l|l|l}
\cline { 2 - 2 } DOI: 10.1159/000438561 & (c) 2015 S. Karger AG, Basel
\end{tabular} \begin{tabular}{l|l} 
and Biochemistry Published online: November 20, 2015 & www.karger.com/cpb
\end{tabular} \\ Wang et al.: Nodal Modulate Differentiation of T-Helper Cells}

Horseradish peroxidase-conjugated secondary antibody and Cy3-labeled secondary antibody were from Boster, Wuhan, China.

\section{Cell culture and treatments}

Human BECs (derived from normal humans) were incubated in Dulbecco's Modified Eagle Medium (DMEM): F12 (1:1) containing $100 \mathrm{IU} / \mathrm{ml}$ penicillin, $100 \mu \mathrm{g} / \mathrm{ml}$ streptomycin, and $10 \%$ heat inactivated fetal bovine serum (FBS) at $37^{\circ} \mathrm{C}$ atmosphere containing $5 \% \mathrm{CO}_{2}$. P. aeruginosa or A. baumanni were heat-killed at $65^{\circ} \mathrm{C}$ for $2 \mathrm{~h}$. After the human BECs were grown in 6 well round-bottom plates with $70 \%$ confluence, BECs were pretreated with $1 \times 10^{6}$ cells of P. aeruginosa or A.baumanni at $37^{\circ} \mathrm{C}$ for additional $48 \mathrm{~h}$.

The strain A2 of human RSV was propagated in HeLa cell monolayer and infected human BECs at multiplicity of infection (MOI) of 0.1 according to experiments in advance. The virus was allowed to incubate for $2 \mathrm{~h}$ at $37^{\circ} \mathrm{C}$ in serum-free DMEM. Then, non-absorbed virus was removed and washed twice, then the cells were cultured in fresh medium with $2 \% \mathrm{FBS}$ for $48 \mathrm{~h}$.

RSV infection assay by real-time PCR and immnofluoresence

RNA of RSV was verified and monitored using a TaqMan®-based real-time PCR kit which includes the reagents for nucleic acid extraction, real-time PCR, high positive, and negative control materials. According to the instructions, the samples were considered negative for RSV when the threshold cycle number (CT) > 32.0. Conservatively, samples with a CT value $\leq 28.9$ were considered clearly positive.

NS1 protein of RSV was assayed by immnofluoresence. BECs were fixed by $95 \%$ ethanol and $0.1 \%$ Triton-X100. The fixed slides were incubated with $3 \% \mathrm{H}_{2} \mathrm{O}_{2}$, and blocked with a normal goat serum for 20 $\mathrm{min}$. The slides were then incubated with a mouse anti-human NS1 monoclonal antibody $(1: 200)$ at $4^{\circ} \mathrm{C}$ for overnight. The Cy3-labeled secondary antibody $(1: 250)$ was added to the slides respectively at $37^{\circ} \mathrm{C}$ for $2 \mathrm{~h}$. The slides were finally mounted with mounting fluid and examined by fluorescence microscopy.

\section{Nodal assay by Western blot}

BECs were washed by ice-cold PBS twice and resolved in RIPA buffer containing $1 \mathrm{mM}$ PMSF, $5 \mathrm{mM}$ $\beta$-glycerophosphate and $1 \%$ of a standard protease inhibitor cocktail (Sigma Chemical Co) on ice for 30 minutes. Insoluble materials were removed by centrifugation for $20 \mathrm{~s}$ at $12,000 \mathrm{~g}$ at $4^{\circ} \mathrm{C}$. The supernatants were collected and the protein concentrations were measured by Bradford method to be adjusted to a final concentration of $10 \mathrm{mg} / \mathrm{ml}$. The supernatants were separated by SDS-PAGE and transferred to nitrocellulose membranes. The membranes were blocked with 3\% BSA in PBS for $2 \mathrm{~h}$ and then incubated with monoclonal rabbit anti-Nodal antibody and appropriate horseradish peroxidase-conjugated secondary antibody. Detection was made using the enhanced chemiluminescene system (Gene Co., Ltd. Hong Kong, China).

\section{Nodal assay by Real-time PCR}

RNAs were isolated from lungs using TRIzol reagent (Invitrogen, USA) according to the instructions of the manufacturer. Each sample was reverse transcribed into cDNA and analyzed by quantitative real-time PCR. Quantitative real-time PCR was performed on "Applied Bio systems Inc 1900 system" using the SYBR Green Real Time PCR Kit (Bio-Rad, America). The primers were 5'-CAT CAT CCG CAG CCT ACA-3' and 5'-CCA TCT TGA AAC CGC TCTAA-3'. Raw data were normalized to GAPDH (5'-CCA CTC CTC CAC CTT TGAC-3' and 5'ACC CTG TTG CTG TAG CCA-3') in each sample. Each cycle included $94^{\circ} \mathrm{C}$ for 30 seconds, $60^{\circ} \mathrm{C}$ for 30 seconds, and $72^{\circ} \mathrm{C}$ for $30 \mathrm{~s}$ after an initial $94^{\circ} \mathrm{C}$ for 4 minutes. Calculate the $\Delta \mathrm{Ct}$ (average $\mathrm{Ct}$ - average $\mathrm{Ct}$ of GAPDH) and $\Delta \Delta \mathrm{Ct}$ (experimental $\Delta \mathrm{Ct}$ - control $\Delta \mathrm{Ct}$ ). Calculate the fold-change from experimental to control as $2^{-\Delta \Delta C \mathrm{Ct}}$. If the fold-change is greater than 1 , then the result may be reported as a fold up-regulation. If the fold-change is less than 1 , then the negative inverse of the result may be reported as a fold down-regulation.

\section{Construction of Nodal-overexpressed and silent plasmids}

To generate Nodal-overexpressed plasmid, the full-length cDNA of Nodal was amplified by PCR, double digested with Xhol I and BamH I (FastDigest, MBI Fermentas, Canada) and cloned into the Xhol I/ BamH I sites of the vector pcDNA3.1(-)-myc-his with T4 DNA Ligase (FastDigest, MBI Fermentas).

To generate the siRNA expression construct of Nodal, three siRNA sequences (GenScript, China) were cloned into the pGCU6/Neo/RFP vector respectively. One of the most effectively silenced plasmids (siRNA sequence for Nodal: AUCUGAAACCGCUCUAAGCAG, 423-445) was chosen for the following studies. 


\section{Cellular Physiology Cell Physiol Biochem 2015;37:2012-2022 \begin{tabular}{l|l|l}
\cline { 2 - 2 } DOI: 10.1159/000438561 & C 2015 S. Karger AG, Basel
\end{tabular} \begin{tabular}{l|l} 
and Biochemistry Published online: November 20, 2015 & www.karger.com/cpb
\end{tabular} \\ Wang et al.: Nodal Modulate Differentiation of T-Helper Cells}

Recombinant plasmid DNA ( $4 \mu \mathrm{g}$ ) and $8 \mu \mathrm{l}$ of the X-treme GENE HP DNA Transfection Reagent (Roche, USA) were mixed with $200 \mu \mathrm{l}$ of medium without antibiotics and FBS and incubated at room temperature for $10 \mathrm{~min}$. Then, this mixture was added to the cells without removing the growth medium. The pcDNA3.1 (-)-myc-his and pGCU6/Neo/RFP vectors were used as vector controls. After $24 \mathrm{~h}$, the transfected cells were selected using G418 (Ceresco, USA) at a concentration of $1000 \mathrm{mg} \mathrm{ml}^{-1}$ and persistently cultured with G418 at a concentration of $200 \mathrm{mg} \mathrm{ml}^{-1}$.

Measurement of cell cycle by flow cytometry

After being cultured in 6-well plates, cells were harvested at a density of 1 x $10^{6} \mathrm{Cells} / \mathrm{mL}$, fixed in cold $70 \%$ ethanol and stored at $-20^{\circ} \mathrm{C}$ overnight. The fixed cells were washed twice with phosphate-buffered saline, stained in a propidium iodide solution (50ug/ml) for $1 \mathrm{~h}$, and treated with a ribonuclease A solution $(20 \mathrm{ug} / \mathrm{ml})$ for 30 minutes. Then Flow cytometry was used to examine cell cycle. Experiments were repeated 4 times.

Co-culture of BECs and $C D 4^{+}$T cells

In co-cultured experiments, BECs were located at the bottom of the culture plate and the Jurkat E 6-1 Cells (CD4 ${ }^{+} \mathrm{T}$ cells strain, a gift from Xiaojian Yao of Manitoba University) were suspended in culture medium. BECs were plated in 6-well culture plates with $2 \times 10^{6}$ cells/well. After BECs were pretreated with P. aeruginosa, A. baumanni or RSV for $24 \mathrm{~h}$, Jurkat E 6-1 Cells were added to the BECs at a ratio of 1:1 so that BECs and lymphocytes were cultured in the same well. After incubation at $37^{\circ} \mathrm{C}$ in $5 \% \mathrm{CO}_{2}$ for $48 \mathrm{~h}$, the lymphocytes were collected.

Flow cytometric analysis of Th subtypes

Jurkat E 6-1 Cells were fixed with 4\% paraformaldehyde for $10 \mathrm{~min}$ at room temperature and permeabilized in permeabilizing solution (eBioscience, USA). After blocking with 3\% BSA for 15 min, cells were stained with appropriate anti-IL-4, IL-17 and IFN-gamma antibodies on ice for 45 min. Isotypematched antibodies were used as controls. The expression levels of antigens were expressed as a percentage of positive cells in the total cells.

Nodal methylation

The Nodal gene sequence was obtained from GenBank. The MethPrimer primer Express software was used to predict the CpG islands of promoter and first exon. Cellular genomic DNA was extracted using the BioTek DNA purification kit (BioTek, Beijing, China). For each sample, a total of 200 ng of genomic DNA from each sample was bisulfate-treated using the methylSEQ ${ }^{\mathrm{TM}}$ Bisulfate Conversion Kit (AB applied biosystems) according to the manufacturer's instructions. The genomic DNA was treated with sodium bisulfate to convert unmethylated cytosines to uracil. Specific primers for methylation measurement were designed using Methyl Primer Express® Software v1.0 as bellows: 5' GGA TTA TTG TTT TTG GGT TTTG 3' and 5' AAA ACC CAA AAC TAC CAC AATT 3' (417 bp). PCR was performed in a thermocycler (Eppendorf Mastercycler gradient, Germany). Mass spectra of PCR products were obtained via MassARRAY Compact MALDI-TOF (Sequenom) and their methylation ratios were generated using the Epityper software version 1.0 (Sequenom).

\section{Statistical Analysis}

Calculations were performed with SPSS software. All values were expressed as Mean \pm SE. Data were compared by use of the Student's $t$-test. $P$ value of less than 0.05 was considered significant.

\section{Results}

Influences of lung microbes on Nodal expression of BECS

Real-time PCR and immunofluorescence showed that the BECs were infected successfully by RSV after $48 \mathrm{~h}$ of incubation (Fig. 1a). Then, we examined the effects of lung microbes on Nodal expression of BECs by using real-time PCR and Western blot. Results of real-time PCR showed that the expression of Nodal mRNA increased by 9.8 folds by RSV infection and 
Fig. 1. (A) RNA and protein expression of RSV in BECs were determined by real time PCR and immunoflurescense $(n=3)$. (B) mRNA expression of Nodal in BECs was determined by real time PCR (n = 3, ** $P<0.01$ versus C). Nodal mRNA increased by 9.8 folds by RSV infection and decreased by 4.14 folds and 2.8 folds by treatment with Paeruginosa or A.baumanni respectively. (C) Protein expression of Nodal in BECs was determined by Western blot (n = 3, ** $P<0.01$ versus $\mathrm{C}$ ). RSV increased the expression of Nodal, while $P$ aeruginosa or A.baumanni inhibited the expression of Nodal. (D) Protein and mRNA expression of Nodal in BECs were determined by Western blot and real time PCR (n $=3$, ** $P<$ 0.01 versus $\mathrm{C} 1$ and \#\# $\mathrm{P}$

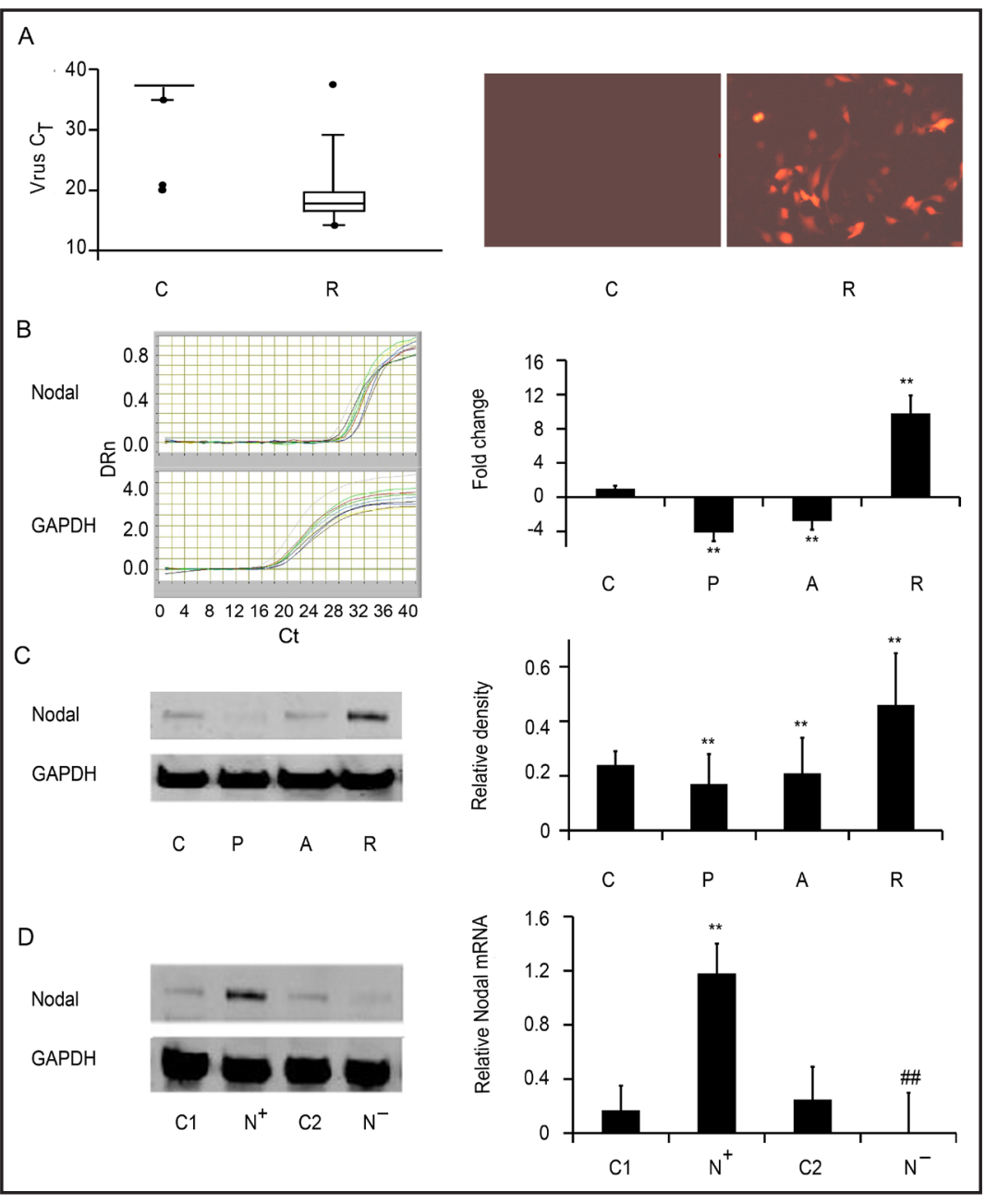

$<0.01$ versus C2). Nodal overexpressed plasmid effectively promoted the expression of Nodal, while Nodal RNAi effectively inhibited the expression of Nodal. (C represents normal BECs; P represents Paeruginosatreated BECs; A represents A.baumanni -treated BECs; R represents RSV-infected BECs; C1 represents pcDNA 3.1-transfected BECs, $\mathrm{N}^{+}$represents Nodal-overexpressed BECs; C2 represents pGCU6-transfected BECs and $\mathrm{N}^{-}$represents Nodal-silent BECs).

decreased by 4.14 folds and 2.8 folds by treatment with Paeruginosa or A.baumanni respectively (Fig. 1b). Results of Western blot also showed that RSV infection promoted the expression of Nodal, while P. aeruginosa or A.baumanni inhibited the expression of Nodal (Fig. 1c). Nodal-overexpressed vector can effectively promote the expression of Nodal, while Nodal RNAi can effectively inhibit mRNA and protein expression of Nodal (Fig. 1d).

Influences of Nodal on cell cycle of BECS

To further examine the role of Nodal in proliferation of BECs, we examined cell cycle of BECs by using flow cytometry. Results showed that P. aeruginosa or A.baumanni significantly promoted the proliferation of BECs, while RSV inhibited the proliferation of BECs (Fig. 2a). Further, in order to determine the effects of Nodal on the proliferation of BECs, we observed that Nodal significantly promoted the proliferation of normal BECs (Fig. 2b). In Nodal-overexpressed BECs, P.aeruginosa or A.baumanni didn't further promote the proliferation of BECs. In Nodal-silent BECs, P.aeruginosa or A.baumanni didn't inhibit the proliferation of BECs, indicating that $P$. aeruginosa or A.baumanni had promoting effect on the proliferation of BECs which is not associated with the expression of Nodal (Fig. 2b). For RSV, in Nodal- 


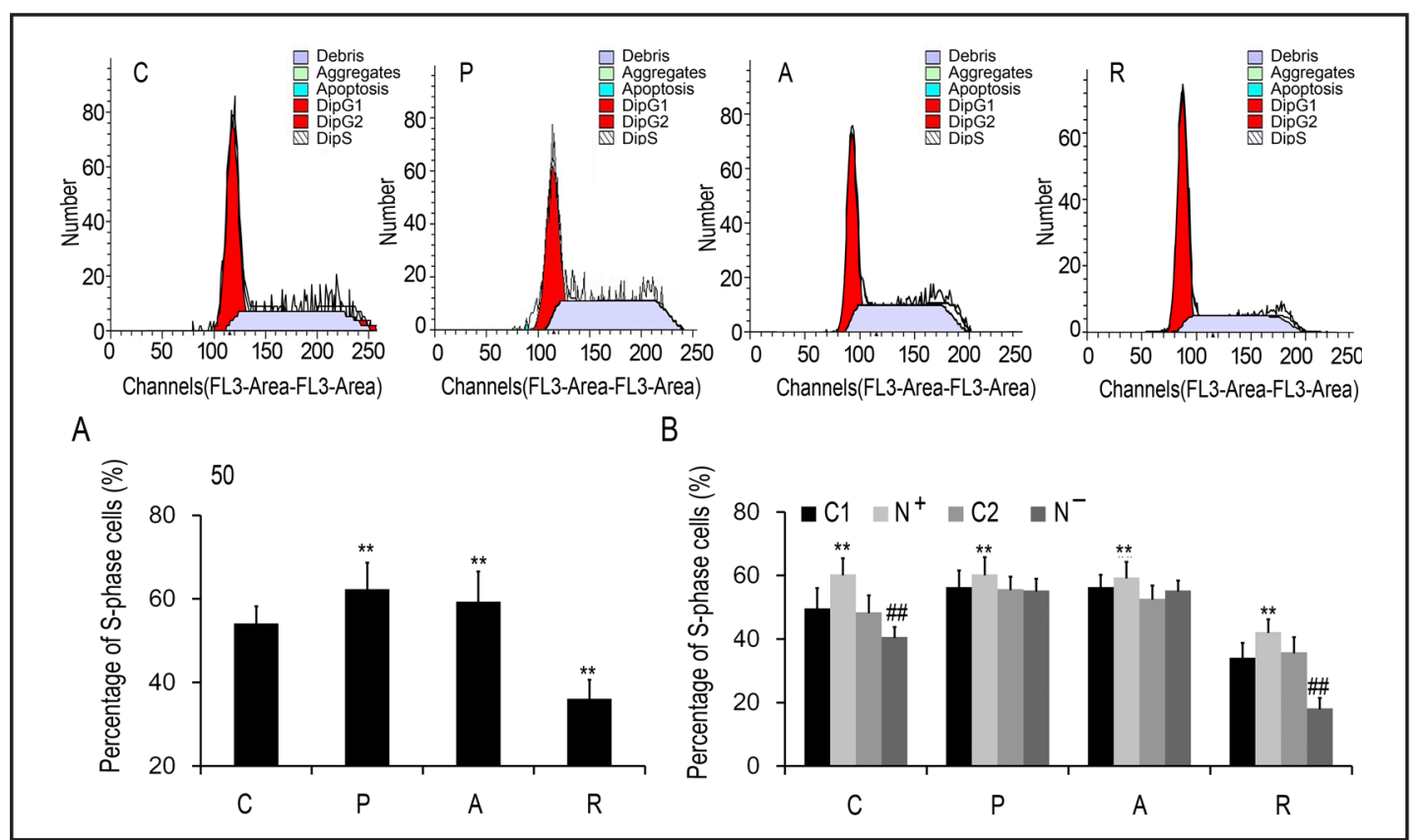

Fig. 2. (A) Effects of lung microbes on proliferation of BECs were assayed by flow cytometry $(n=4$, ** $P<0.01$ versus C). P. aeruginosa or A.baumanni significantly promoted the proliferation of BECs, while RSV inhibited the proliferation of BECs. (B) Effects of Nodal on proliferation of BECs were assayed by flow cytometry ( $\mathrm{n}=4,{ }^{* *} P<0.01$ versus $\mathrm{C} 1$ and ${ }^{\# \#} P<0.01$ versus $\mathrm{C} 2$ ). Nodal promoted the proliferation of normal BECs P.aeruginosa or A.baumanni significantly promoted the proliferation of Nodal-overexpressed BECs, but had no effects on Nodal-silent BECs when compared with corresponding controls. RSV significantly promoted the proliferation of Nodal-over expressed BECs and inhibited the proliferation of Nodal-silent BECs.

overexpressed BECs, RSV promoted the proliferation of BECs and in Nodal-silent BECs, RSV inhibited the proliferation BECs, indicating that the promoting activities of RSV on proliferation of BECs were associated with the expression of Nodal (Fig. 2b).

Influences of Nodal on the differentiation of Th subsets derived by BECs

To further examine the role of Nodal expressed in the BECs in the differentiation of Th subsets, we examined the distribution of Th subsets after co-culture with BECs. As expected, P. aeruginosa or A.baumanni-treated BECs stimulated differentiation of Th1, while RSV-infected BECs promoted the differentiation of Th2 and Th17 (Fig. 3). Further, we observed that Nodal-overexpressed BECs or Nodal-silent BECs had no effects on the differentiation of Th subsets (data not shown); however, Th2 and Th17 differentiation was induced, while IFN-gamma differentiation was suppressed in Nodal-overexpressed BECs after treatment wth P. aeruginosa, A.baumanni or RSV. Nodal RNAi promoted the differentiation of Th1, and inhibited the differentiation of Th2 and Th17 after treatment wth P. aeruginosa, A.baumanni or RSV (Fig. 4).

Methylation in Nodal promoter influenced by lung microbes.

Human Nodal sequence was searched in GenBank. The length of CpG islands was $2000 \mathrm{bp}$ ( $1 \mathrm{bp}$ to $2000 \mathrm{bp}$ ). We examined 496bp to 912bp (32 CpG sites) containing the whole promoter and the first exon. Agarose gel electrophoresis of bisulfate sequencing PCR amplification products showed a single desired product of $417 \mathrm{bp}$ as the expected target fragment. MALDI-TOF MS analyzer detected that CpG islands in Nodal promoter showed a hyper-methylation in normal BECs. The average methylation rate of $\mathrm{CpG}$ island increased with treatment of $P$. aeruginosa or A.baumanni ( $\mathrm{P}=0.0045$ and $\mathrm{P}=0.0054)$ while decreased with treatment of RSV (P=0.0037, Fig. 5). 
A
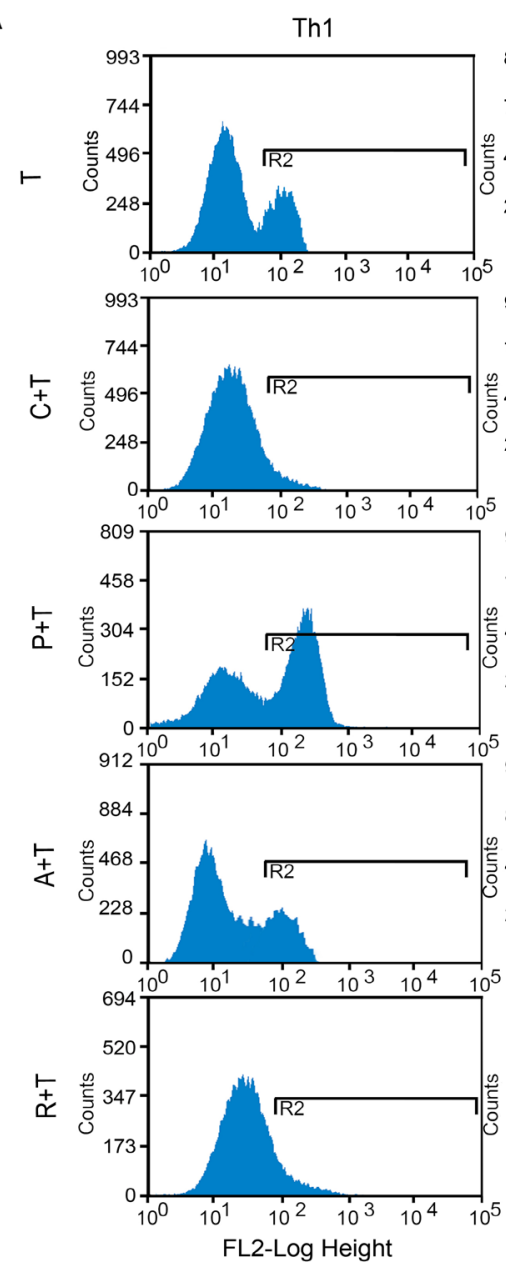

B
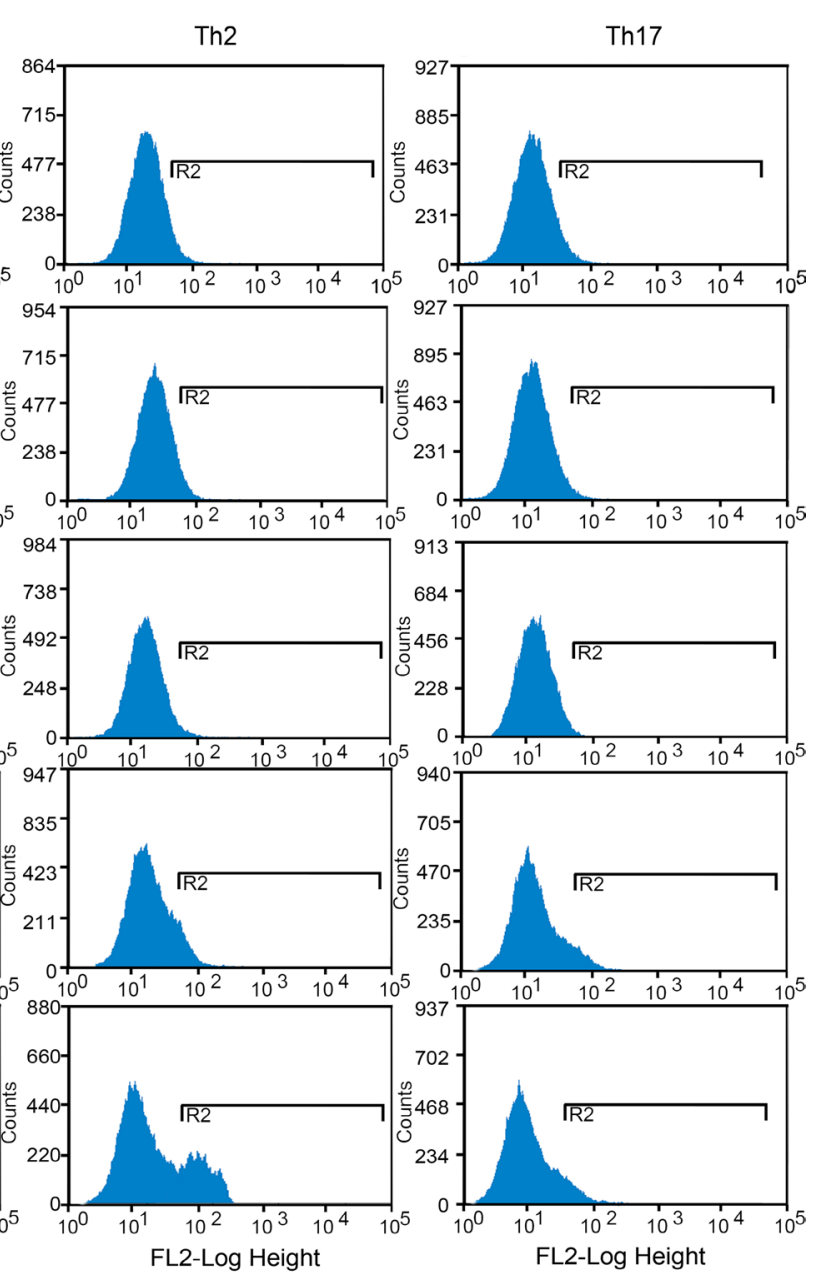

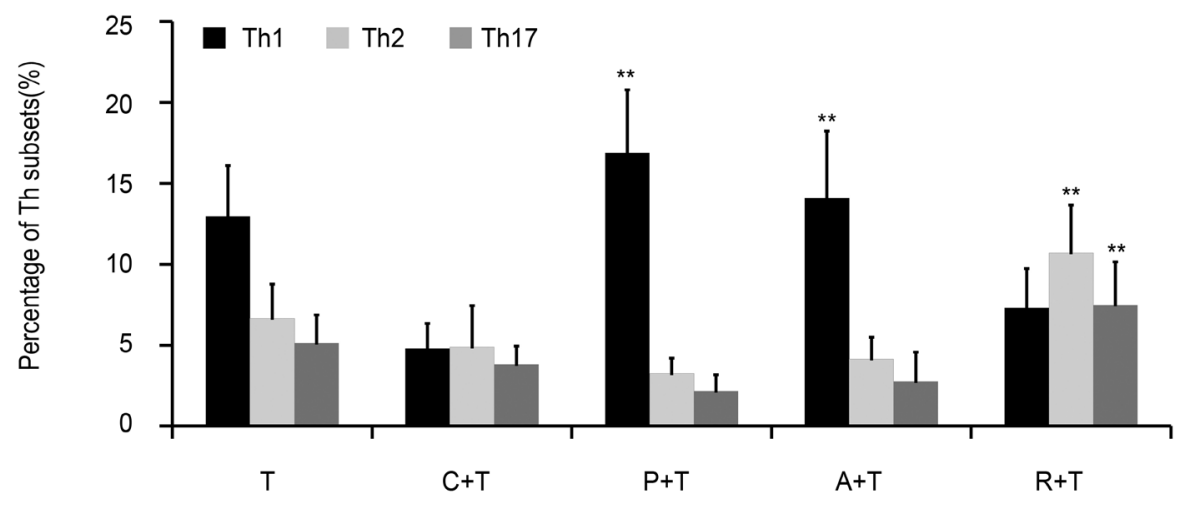

Fig. 3. Effects of lung microbes on differentiation of Th cells were assayed by flow cytometry $(n=4$, ** $\mathrm{P}<0.01$ versus $\mathrm{C}+\mathrm{T}$ group). P. aeruginosa or A.baumanni-treated BECs stimulated differentiation of Th1cells, while RSV-infected BECs promoted the differentiation of Th2 and Th17 cells. (T represents CD4 ${ }^{+} \mathrm{T}$ cells, others are same with Fig. 1).

\section{Discussion}

The previous studies of other teams and our team showed that defect in function and integrity of airway epithelia had consistency in the vast majority of patients with AHR and could explain many important clinical pathological features of these patients $[20,21]$.

\section{KARGER}


Fig. 4. Effects of Nodal on differentiation of Th cells cocultured with $P$. aeruginosa (A), A.baumanni (B) or RSV-treated BECs (C) were assayed by flow cytometry (n $=4$, ${ }^{* *} P<0.01$ versus $\mathrm{C} 1+\mathrm{T}$; \# \# $P<$ 0.01 versus $\mathrm{C} 2+\mathrm{T}$ ). Differentiation of Th2 and Th17 was induced, while differentiation of IFN-gamma was suppressed in Nodal-overexpressed BECs after treatment with $P$ aeruginosa, A.baumanni or RSV. Nodal-silent BECs promoted the differentiation of Th1, and inhibit the differentiation of Th2 and Th17 after treatment with P. aeruginosa, A.baumanni or RSV.

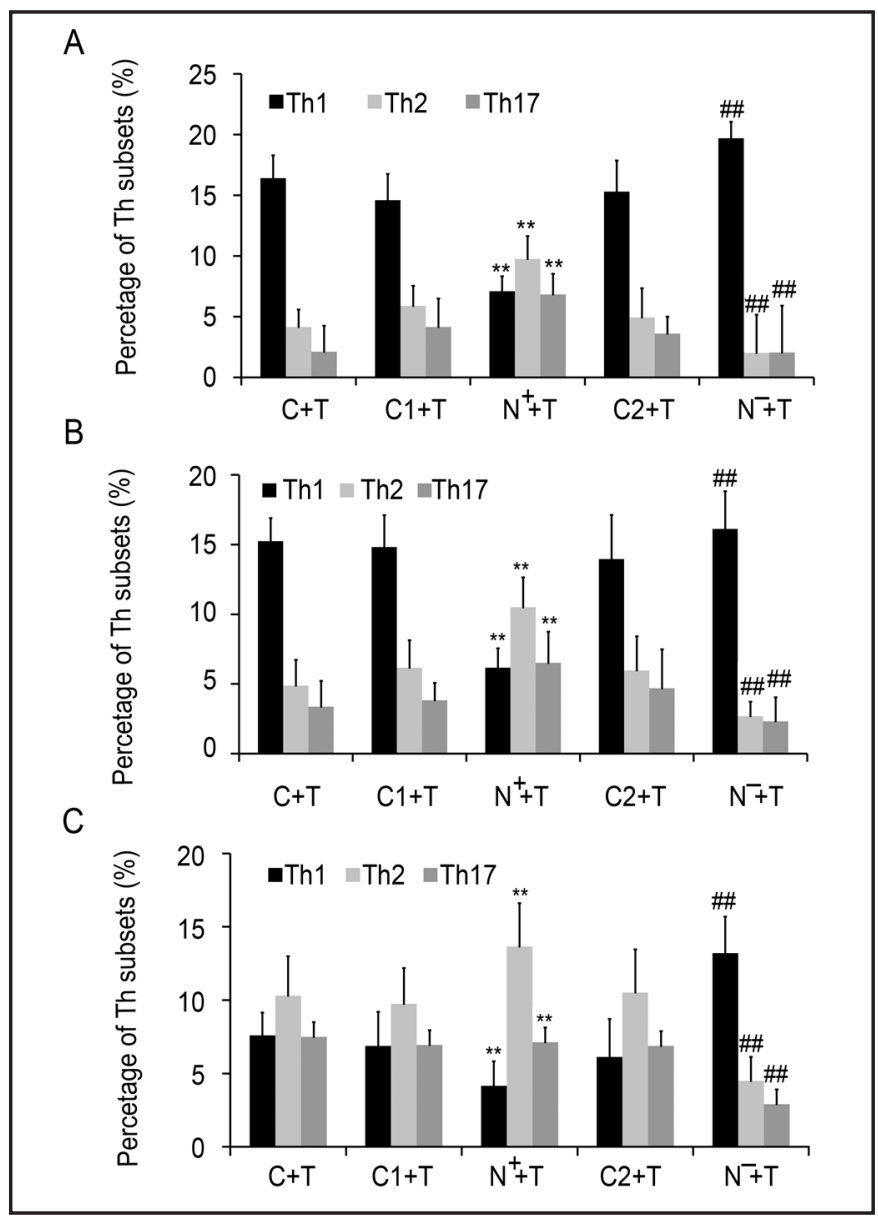

Fig. 5. Quantitative DNA methylation of CpG islands in Nodal promoter region was assayed by sequencing ( $\mathrm{n}=3,{ }^{* *} P<$ 0.01 versus $C$ ). The average methylation rate of $\mathrm{CpG}$ islands increased with treatment of $P$. aeruginosa or A.baumanni while decreased with treatment of RSV.

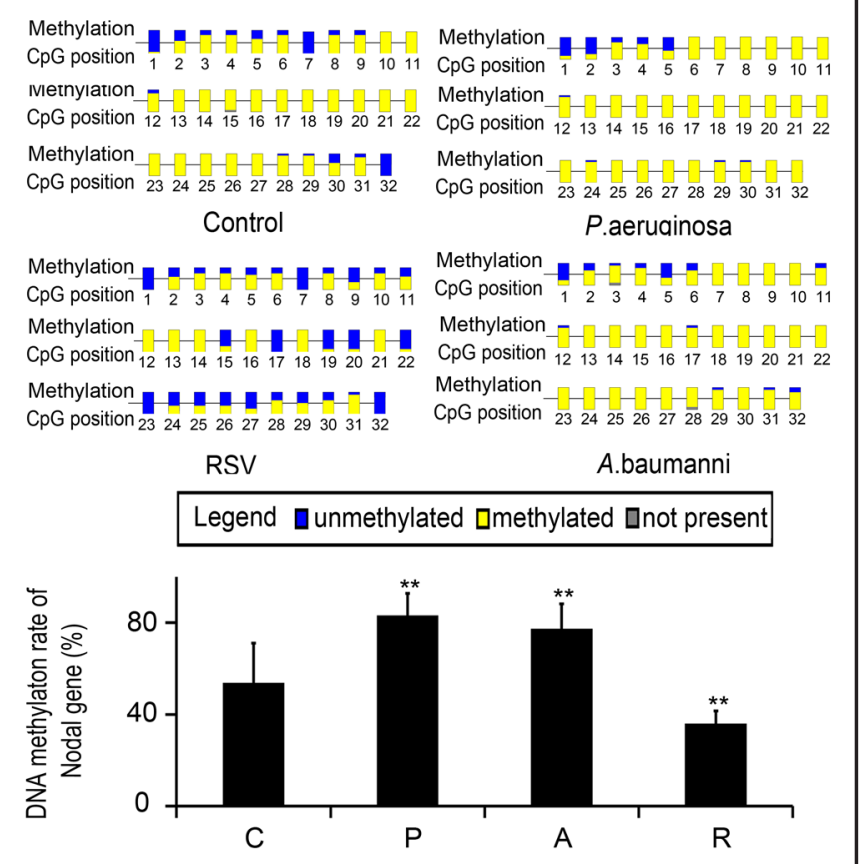

Nodal is one of important genes during embryonic development, and remains a low level of expression in adults which may ascribe to hypermethylation in the promoter induced by some microbes such as P. aeruginosa or A.baumanni. However, some factors such as 
Fig. 6. Effects of $P$. aeruginosa, A.baumanni or RSV on proliferation of BECs. P. aeruginosa or A.baumanni promoted proliferation and wound repair of BECs through TLR4 signaling, but inhibited proliferation and differentiation of BECs through Nodal signaling. However, RSV inhibited the proliferation of BECs through apoptosis caused by TNF signaling and promoted the proliferation and differentiation of BECs through Nodal signaling.

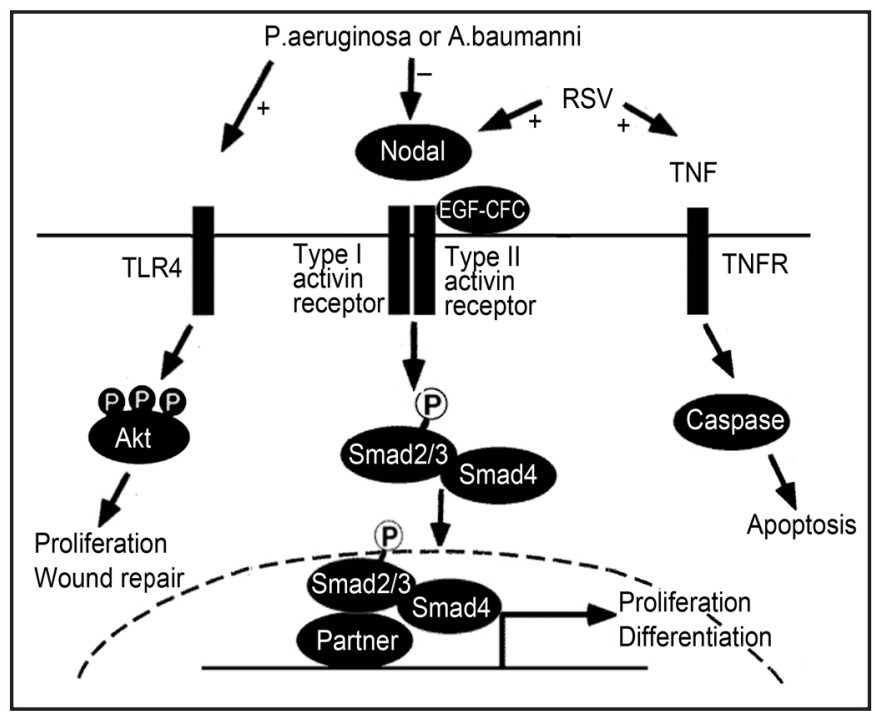

RSV infection may lead to demethylation of promoter and high level of expression of Nodal in adults.

After activation and expression, Nodal significantly promoted the proliferation of BECs according to our results. However, P. aeruginosa or A.baumanni also significantly promoted the proliferation of BECs, while they inhibited the expression of Nodal. This discrepancy maybe ascribe to the effects that $P$. aeruginosa or A.baumanni promoted the proliferation of BECs through other signaling such as TLR-4 or TLR-5 signaling, not Nodal signaling [22, 23]. In previous study, we confirmed that $P$. aeruginosa promoted the proliferation and wound repair of normal, OVA-stressed or RSV-infected BECs through TLR-4 and TLR-5 signaling [8, 9]. On the other hand, RSV inhibited the proliferation of BECs, while RSV promoted the expression of Nodal. This discrepancy maybe ascribe to the effects that RSV promoted apoptosis of BECs, which belied the effect of Nodal (Fig. 6) [24].

Further studies showed that expression levels of Nodal on BECs were associated with the differentiation of Th subsets. Nodal-overexpressed BECs had no effects on the differentiation of Th at resting conditions; however, after the stimulation of antigens such as $P$. aeruginosa, A.baumanni or RSV, they promoted the differentiation of Th2 and Th17, and inhibited the differentiation of Th1. On the contrary, Nodal-silent BECs, after the stimulation of antigens such as P. aeruginosa, A.baumanni or RSV, promoted differentiation of Th1 and inhibited differentiation of Th2 and Th17. That P. aeruginosa and A.baumanni promoted the differentiation of Th2 and Th17, and inhibited the differentiation of Th1 and RSV had the opposite effects was associated with the expression levels of Nodal. The differentiation of Th2 and Th17 are closely associated with the occurrence and development of many diseases such as atherosclerosis and atherosclerotic cerebral infarction [25-27].

In conclusion, our data showed that expression levels of Nodal were influenced by lung microbes. Nodal promoted proliferation of BECs and subsequent promoting differentiation of Th2 and Th17 and inhibiting differentiation of Th1 which may cause imbalance of airway microenvironment. P. aeruginosa or A.baumanni may be as a strategy for the treatment and prevention of AHR through inhibition of Nodal induced immune imbalance.

\section{Acknowledgements}

This work was supported by grant \# 13K001 (Yurong Tan) from University Innovation Platform Foundation of Hunan province and grant\#2013JSJJ040(LiliWang) and \#2013JSJJ059 (Yurong Tan) from Teacher Funds of Central South University. 


\section{Cellular Physiology Cell Physiol Biochem 2015;37:2012-2022 \begin{tabular}{l|l|l}
\hline DOI: 10.1159/000438561 & (C) 2015 S. Karger AG, Basel
\end{tabular} www.karger.com/cpb

\section{Disclosure Statement}

None.

\section{References}

1 Marshall GD Jr, Agarwal SK: Stress, immune regulation, and immunity: applications for asthma. Allergy Asthma Proc 2000;21:241-246.

2 Dinh QT, Suhling H, Fischer A, Braun A, Welte T: [Innervation of the airways in asthma bronchiale and chronic obstructive pulmonary disease (COPD)]. Pneumologie 2011;65:283-292.

3 Dicpinigaitis P: Effect of viral upper respiratory tract infection on cough reflex sensitivity. J Thorac Dis 2014;6:S708-711.

4 Royce SG, Li X, Tortorella S, Goodings L, Chow BS, Giraud AS, Tang ML, Samuel CS: Mechanistic insights into the contribution of epithelial damage to airway remodeling. Novel therapeutic targets for asthma. Am J Respir Cell Mol Biol 2014;50:180-192.

5 Malmström K, Pelkonen AS, Mäkelä MJ: Remodeling, inflammation and airway responsiveness in early childhood asthma.Curr Opin Allergy Clin Immunol 2013;13:203-210

6 Willner DL, Hugenholtz P, Yerkovich ST, Tan ME, Daly JN, Lachner N, Hopkins PM, Chambers DC: Reestablishment of recipient-associated microbiota in the lung allograft is linked to reduced risk of bronchiolitis obliterans syndrome.Syndrome. Am J Respir Crit Care Med 2013;187:640-647.

7 Venkataraman A, Bassis CM, Beck JM, Young VB, Curtis JL, Huffnagle GB, Schmidt TM: Application of a neutral community model to assess structuring of the human lung microbiome. MBio 2015;6:e02284-14.

8 Tan Y, Liu H, Yang H, Wang L, Qin X: An inactivated Pseudomonas aeruginosa medicament inhibits airway allergic inflammation and improves epithelial functions. J Physiol Sci 2013;63:63-69.

9 Wang LL, Qin L, Yang HH, Peng D, Ma QS, Wu GJ, Liu SP, Tan YR, Qin XQ: An Inactivated P. aeruginosa Immunomodulator Restores Imbalanced Epithelial Function Induced by In Vitro RSV Persistent Infection. J Allergy Ther 2013;4:147.

10 Tan YR, Peng D, Chen CM, Qin XQ: Nonstructural protein-1 of respiratory syncytial virus regulates HOX gene expression through interacting with histone. Mol Biol Rep 2013;40:675-679.

11 Robertson EJ: Dose-dependent Nodal/Smad signals pattern the early mouse embryo. Semin Cell Dev Biol 2014;32:73-79.

12 Molina MD, de Crozé N, Haillot E, Lepage T: Nodal: master and commander of the dorsal-ventral and leftright axes in the sea urchin embryo. Curr Opin Genet Dev 2013;23:445-453.

13 Quail DF, Siegers GM, Jewer M, Postovit LM: Nodal signalling in embryogenesis and tumourigenesis. Int J Biochem Cell Biol 2013;45:885-898.

14 Vallier L, Alexander M, Pedersen RA: Activin/Nodal and FGF pathways cooperate to maintain pluripotency of human embryonic stem cells. J Cell Sci 2005;118:4495-4509.

15 Katsu K, Tatsumi N, Niki D, Yamamura K, Yokouchi Y: Multi-modal effects of BMP signaling on Nodal expression in the lateral plate mesoderm during left-right axis formation in the chick embryo. Dev Biol 2013;374:71-84.

16 Calvanese L, Sandomenico A, Caporale A, Focà A, Focà G, D'Auria G, Falcigno L, Ruvo M: Conformational features and binding affinities to Cripto, ALK7 and ALK4 of Nodal synthetic fragments. J Pept Sci 2015;21:283-293.

17 Lawrence MG, Margaryan NV, Loessner D, Collins A, Kerr KM, Turner M, Seftor EA, Stephens CR, Lai J, APC BioResource, Postovit LM, Clements JA, Hendrix MJ: Reactivation of embryonic nodal signaling is associated. Prostate 2011;71:1198-1209.

18 Kong B, Wang W, Esposito I, Friess H, Michalski CW, Kleeff J: Increased expression of Nodal correlates with reduced patient survival in pancreatic cancer. Pancreatology 2015; 15:156-161.

19 Cavallari C, Fonsato V, Herrera MB, Bruno S, Tetta C, Camussi G: Role of Lefty in the anti tumor activity of human adult liver stem cells. Oncogene 2013;32:819-826.

20 Hérard AL, Pierrot D, Hinnrasky J, Kaplan H, Sheppard D, Puchelle E, Zahm JM: Fibronectin and its alpha 5 beta 1-integrin receptor are involved in the wound-repair process of airway epithelium. Am J Physiol 1996;271:L726-733. 


\section{Cellular Physiology Cell Physiol Biochem 2015;37:2012-2022}

and Biochemistry

21 Cheng D, Xue Z, Yi L, Shi H, Zhang K, Huo X, Bonser LR, Zhao J, Xu Y, Erle DJ, Zhen G: Epithelial interleukin-25 is a key mediator in Th2-high, corticosteroid-responsive asthma. Am J Respir Crit Care Med 2014;190:639-648.

22 Adamo R, Sokol S, Soong G, Gomez MI, Prince A: Pseudomonas aeruginosa flagella activate airway epithelial cells through asialoGM1 and toll-like receptor 2 as well as toll-like receptor 5. Am J Respir Cell Mol Biol 2004;30:627-634.

23 Koff JL, Shao MX, Kim S, Ueki IF, Nadel JA: Pseudomonas lipopolysaccharide accelerates wound repair via activation of a novel epithelial cell signaling cascade. J Immunol 2006;177:8693-8700.

24 Kotelkin A, Prikhod'ko EA, Cohen JI, Collins PL, Bukreyev A: Respiratory syncytial virus infection sensitizes cells to apoptosis mediated by tumor necrosis factor-relatedapoptosis-inducing ligand. J Virol 2003;77:9156-9172.

25 Li Q Wang Y, Li H, Shen G, Hu S: Ox-LDL influences peripheral Th17/Treg balance by modulating Treg apoptosis and Th17 proliferation in atherosclerotic cerebral infarction. Cell Physiol Biochem 2014;33:1849-1862.

26 Yu M, Hu J, Zhu MX, Zhao T, Liang W, Wen S, Li HH, Long Q Wang M, Guo HP, Cheng X, Liao YH, Yuan J: Cardiac fibroblasts recruit Th17 cells infiltration into myocardium by secreting CCL20 in CVB3-induced acute viral myocarditis. Cell Physiol Biochem 2013;32:1437-1450.

27 Lin J, Chang W, Dong J, Zhang F, Mohabeer N, Kushwaha KK, Wang L, Su Y, Fang H, Li D: Thymic stromal lymphopoietin over-expressed in human atherosclerosis: potential role in Th17 differentiation. Cell Physiol Biochem. 2013;31:305-318. 